Katarina Gospodarič

Šolski center Postojna

\title{
LA ENSEÑANZA DE LA PRONUNCIACIÓN DEL ESPAÑOL COMO LENGUA EXTRANJERA PARA ESLOVENOHABLANTES: PUNTO DE PARTIDA
}

\section{Introducción}

¿Enseñar la pronunciación o no? ¿Y cómo hacerlo? La pregunta intrigante cuyas respuestas han determinado la posición de la pronunciación dentro de los diferentes métodos y enfoques de la enseñanza de lenguas extranjeras: desde concederle la máxima importancia con el movimiento reformista hasta caer casi en el olvido con la introducción del enfoque comunicativo, desde la descripción exhaustiva del aparato fono-articulatorio hasta la simple imitación traspasando toda la responsabilidad del aprendizaje al alumno y su propia competencia. Esta búsqueda de un papel que corresponda adecuadamente a la pronunciación en la enseñanza y el proceso del aprendizaje de LE, se debe también a escasa indagación en el campo de la adquisición de la pronunciación, sobre todo de la prosodia. En la actualidad, todo esto resulta en carente formación del profesorado, recursos y materiales didácticos poco adecuados y la opinión prevaleciente, tanto entre profesores como alumnos, que tiende a relegar la pronunciación al segundo plano. Se observa este problema, sobre todo, cuando se pretende impartir clases de pronunciación desde el punto de vista del enfoque comunicativo porque los especialistas no han dado muchas respuestas a cómo diseñar actividades de pronunciación comunicativas y dónde se encuentra la pronunciación en comparación con otros conocimientos de la lengua dentro de este enfoque.

Sin embargo, los expertos están de acuerdo en que la adquisición de la pronunciación de una lengua extranjera es uno de los conocimientos de la lengua que más se ve influido por los rasgos fonéticos y fonológicos de la lengua materna del hablante, su edad y las situaciones psicológicas, sociológicas y físicas en las que se encuentra. Tampoco no cabe duda de que la adquisición de la fonología difiere cualitativamente de la adquisición del léxico o la sintaxis. Por ejemplo, hay alumnos jóvenes que dominan perfectamente la pronunciación pero tienen vacíos graves en la gramática o el léxico. Por otro lado, hay adultos que consiguen tener un buen dominio de la sintaxis y del léxico pero tienen claros problemas con la pronunciación. Otro factor importante es la adquisición y el aprendizaje de la prosodia (Nuefeld, 1987) que es tanto o más importante que la de los sonidos porque las desviaciones de las características prosódicas influyen más en la formación del acento extranjero y en la inteligibilidad que la incorrecta pronunciación de los sonidos (Anderson-Hsieh et al., 1992). Además, los estudios de Munro y Dewing (1999) han demostrado que no deben confundirse inteligibilidad y comprensibilidad con acento extranjero ya que el habla con un fuerte acento extranjero no es necesariamente ininteligible y viceversa.

El papel de la instrucción explícita en el proceso del aprendizaje es otra cuestión bastante discutida entre los expertos y los mismos profesores, lo que, en muchos casos, resulta en evitar impartirla en clase. Sin embargo, se ha comprobado que, no sólo la exposición extensiva al input auditivo, sino también la instrucción fonética y fonológica contribuyen a mejorar la pronunciación de un individuo (Elliott, 1995, Castino, 1996, Cortés, 1992, Der- 
wing et al., 1998) y debe empezar desde los primeros días de clase. Los objetivos del proceso instructivo tienen que tener en cuenta tanto la inteligibilidad y fluidez como la corrección fónica para disminuir el efecto del acento extranjero, dependiendo de las necesidades de los alumnos y sus objetivos personales.

Para definir concretamente los objetivos del proceso instructivo es necesarios saber cuáles son los rasgos de la pronunciación de la lengua extranjera que representan cierta dificultad para un hablante no nativo. Por eso, deben realizarse ciertas pruebas auditivas de percepción, ya que no cabe duda que la incorrecta percepción condiciona la incorrecta producción. En cuanto a los hablantes eslovenos, hasta ahora, no se han tenido al alcance ningunos datos empíricos para poder concretar estos objetivos. A consecuencia, se han realizado las pruebas auditivas de percepción cuyo propósito ha sido recoger los datos sobre los rasgos segmentales y suprasegmentales que a hablantes eslovenos les resultan difíciles de percibir correctamente, y así tener un punto de partida para concretar los contenidos de la pronunciación para la enseñanza de eslovenohablantes y para diseñar las actividades.

\section{Pruebas auditivas de percepción del español para eslovenohablantes}

\subsection{Realización de las pruebas}

Las pruebas de percepción contienen la prueba de percepción de los sonidos, de la acentuación y de la entonación.

Para la prueba de percepción de los sonidos se han elegido 80 pares mínimos que se diferencian en un sólo fonema. Los oyentes ${ }^{1}$ han escuchado la serie de tres palabras en las que una es diferente a las otras dos, por ejemplo AAB o ABA, y debía ser identificada. Para los pares mínimos se han elegido todos los fonemas del español repitiendo más veces los que, según la comparación de los dos sistemas fonético-fonológicos esloveno y español y la experiencia docente personal, representarían mayor dificultad para los oyentes.

Para la prueba de percepción de la acentuación se ha elegido la propuesta de Cortés (2002:153-154): una lista de 35 palabras bisílabas y trisílabas combinando todos los patrones acentuales posibles. Para realizar la tarea, los oyentes tenían que identificar la sílaba fuerte de cada palabra.

La prueba de la percepción de la entonación también se basa en la propuesta de Cortés (2002:155-157), que contiene una lista de 24 enunciados de los cuales seis son declarativos, seis enfáticos, seis preguntas absolutas y seis preguntas pronominales. Los enunciados se repiten una vez alterando el orden. Los oyentes tenían que marcar si el enunciado escuchado es declarativo, interrogativo o enfático.

Los informantes ${ }^{2}$ de todas las pruebas han sido las personas cuya lengua materna es español, la variante castellana-peninsular, alternando las voces femeninas y masculinas en cada una de las pruebas.

En las pruebas han participado los siguientes grupos de oyentes:

GRUPO A: 61 oyentes (59 en la prueba de percepción de los sonidos porque se han descartado 2 hojas de respuestas por ser rellenadas incorrectamente) de 14 a 63 años de edad sin cualquier conocimiento previo de español.

\footnotetext{
1 Se llama oyentes a los que han escuchado y realizado las pruebas.

2 Se llama informantes a los que han dado su voz para la grabación.
} 
GRUPO B: 40 estudiantes del español de los cuales 21 estudiantes han sido del primer año con 54 horas de clase impartida - grupo B1, 10 del segundo año con 120 horas de clase - grupo B2 y 9 del tercer año con 200 horas de clase - grupo B3, para poder contrastar los conocimientos de la pronunciación y comprobar la eficacia del proceso instructivo.

Todos los oyentes viven en la parte oeste de Eslovenia donde se observa la influencia del italiano en el acento regional.

Las pruebas se han realizado en un aula con el CD radiocasete y los oyentes han escuchado las pruebas una sola vez. Sin lugar a dudas, los resultados de las pruebas serían más fiables si se hubieran llevado a cabo en el laboratorio de idiomas y grabado por locutores profesionales en un estudio. Lamentablemente, no se han tenido al alcance estos recursos técnicos y tampoco los materiales.

\subsection{Los resultados}

\section{Los sonidos}

Las figuras 1, 2 y 3 muestran los procentajes de la identificación errónea de las vocales, las consonantes y en total de los dos grupos.

Según los resultados, los eslovenohablantes no tienen mucha dificultad en identificar correctamente los sonidos españoles. Los resultados del grupo A muestran que, de las vocales, destacan las identificaciones erróneas entre [o] y [u] $(0,1 \%)$, y [o] y [uo] $(0,2 \%)$; entre [e] y [a] en las terminaciones de plural $(0,4 \%)$ y los triptongos [iei] - [iai] $(0,1 \%)$. En cuanto a las consonantes hay más errores. El porcentaje más alto de identificaciones erróneas se observa para las consonantes [ひ] con la combinación [ni] $(1,3 \%)$, entre [p] y [ ] $(0,8 \%)$, el grupo consonántico [pt] y [kt] $(0,8 \%)$. Siguen los errores de identificación con el $0,4 \%$ entre [s] y [T], [f] y [T], [ $\Lambda$ ] y [x] y entre [L] y [li]. Con el $0,3 \%$ se encuentra $[\mathrm{B}]$ y $[\Gamma] ;[\Delta]$ y $[\Gamma]$. Con el $0,2 \%$ de todos los errores tenemos $[\mathrm{r}]$ y

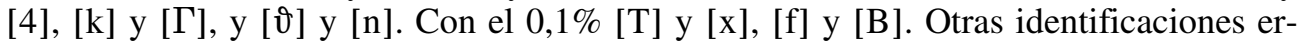
róneas llegan al porcentaje tan bajo que no representan una dificultad significativa.

En resumen, se puede concluir que las identificaciones erróneas con los porcentajes más altos incluyen los sonidos que no existen en el esloveno: [ $, \mathrm{B}, \mathrm{T}, \Gamma, \Lambda, \Delta, \mathrm{r}, \mathrm{x}]$. Sorprende el porcentaje bastante alto de identificación correcta entre los fonemas $[4, \mathrm{r}]$ aunque en la producción casi nunca se realiza la vibrante múltiple, ya que no existe en el esloveno.

Los resultados del grupo B no difieren mucho, el porcentaje de error total es el 7,1\% (grupo B1 6,5\%, B2 8,5\% y B3 7\%). Sorprende el porcentaje de error muy bajo del grupo B1. Probablemente, se puede explicar este resultado por el proceso instructivo porque la prueba auditiva se realizó sólo unas semanas después de que los oyentes hubieran realizado una presentación de lectura. Por otro lado, el grupo B2 ha cometido incluso más errores que el grupo A. Como se trata de un grupo pequeño de 10 oyentes y de la diferencia de un sólo $0,5 \%$, es probablemente difícil hablar de un resultado muy significativo. 


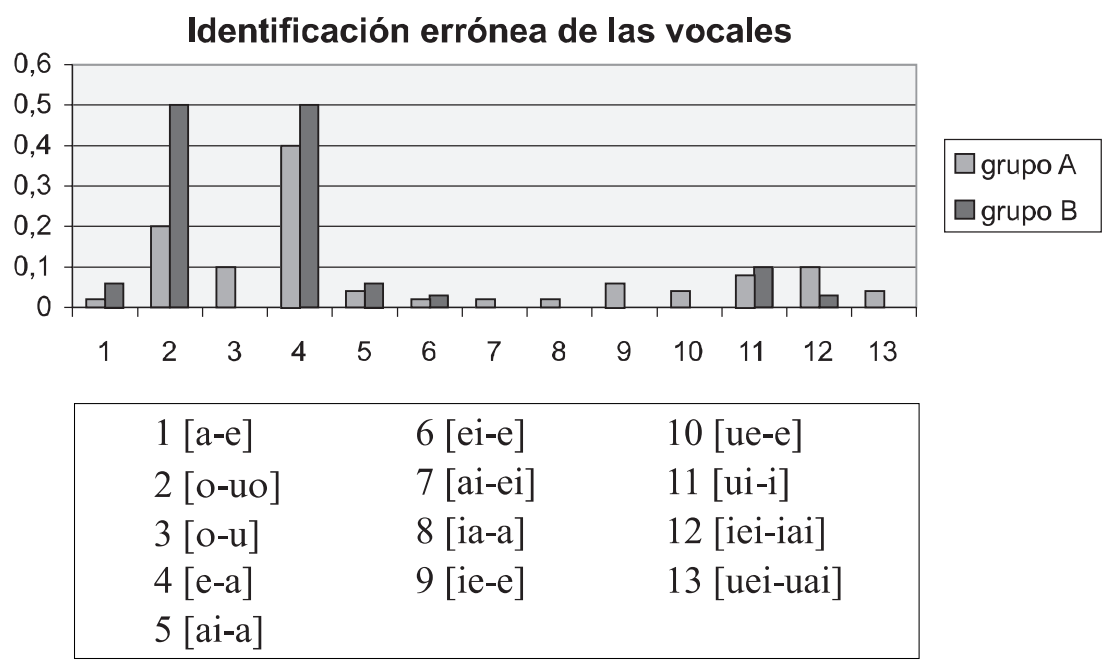

Figura 1: Los porcentajes de la identificación errónea de las vocales

Identificación errónea de las consonantes

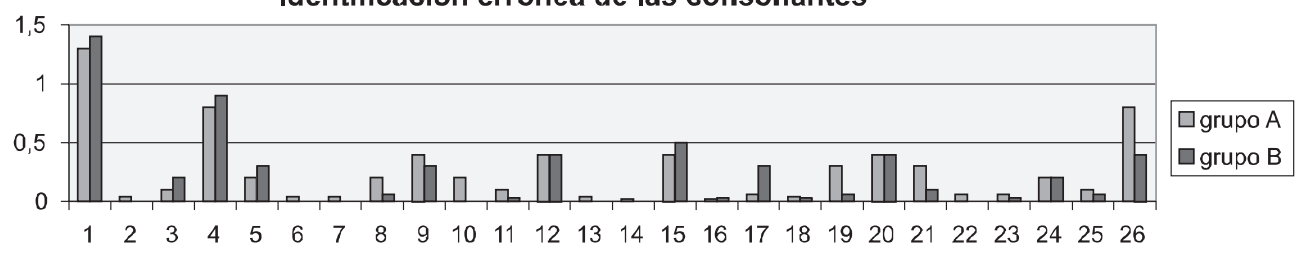

\begin{tabular}{|c|c|c|c|c|c|}
\hline 1 [ni-n] & $6[t-ð]$ & $11[f-\beta]$ & $15[\gamma-x]$ & $19[\beta-\gamma]$ & 23 [m-n] \\
\hline $2[b-p]$ & $7[\mathrm{k}-\mathrm{g}]$ & $12[\mathrm{f}-\boldsymbol{e}]$ & $16[\partial-\Theta]$ & $20[\Lambda-\mathrm{li}]$ & $24[n-n]$ \\
\hline $3[\theta-x]$ & $8[k-\gamma]$ & $13[\beta-ð]$ & $17[\Lambda-j]$ & 21 [ठ઼-ช] & 25 [ere-eer] \\
\hline $4[p-\beta]$ & $9[s-\Theta]$ & $14[\theta-\mathrm{t}]$ & $18[\Lambda-I]$ & $22[\mathrm{x}-\mathrm{g}]$ & $26[\mathrm{pt}-\mathrm{kt}]$ \\
\hline $5[r-r]$ & $10[f-p]$ & & & & \\
\hline
\end{tabular}

Figura 2: Los porcentajes de la identificación errónea de las consonantes

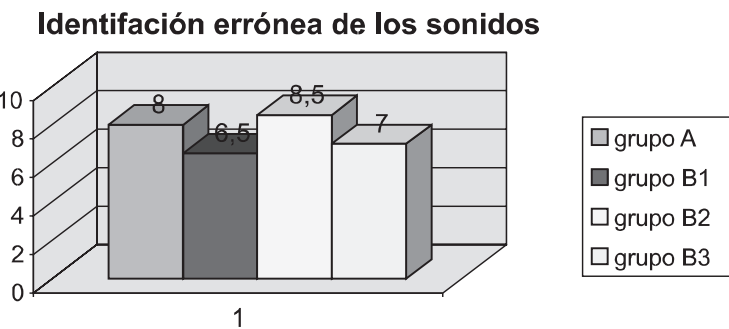

Figura 3: Comparación de los resultados de la percepción de los sonidos entre el grupo A y B 


\section{La acentuación}

A continuación se presentan los resultados de la percepción de la acentuación, divididos en la identificación errónes de las bisílabas (fig. 4) y trisílabas (fig.5 ), y la comparación de los resultados entre el grupo A y B (fig. 6).

Con las palabras bisílabas se han cometido casi dos veces más errores con las palabras agudas que con las llanas. Al contrario, con las trisílabas, el porcentaje más alto de error se encuentra con las palabras llanas y esdrújulas; y el más bajo, con agudas. Además, según el tipo de error de identificación, las llanas se confunden más frecuentemente con las esdrújulas y las esdrújulas, con las llanas. Comparando los resultados del grupo A con el grupo B, el porcentaje de error ha descendido en mayor grado con las trisílabas cuando se identifican las llanas como esdrújulas y viceversa. Lo que menos ha mejorado han sido la identificación correcta de las bisílabas y trisílabas agudas, lo que se puede explicar con que en esloveno el acento suele caer sobre la penúltima sílaba y hay pocas palabras agudas, sobre todo bisílabas.

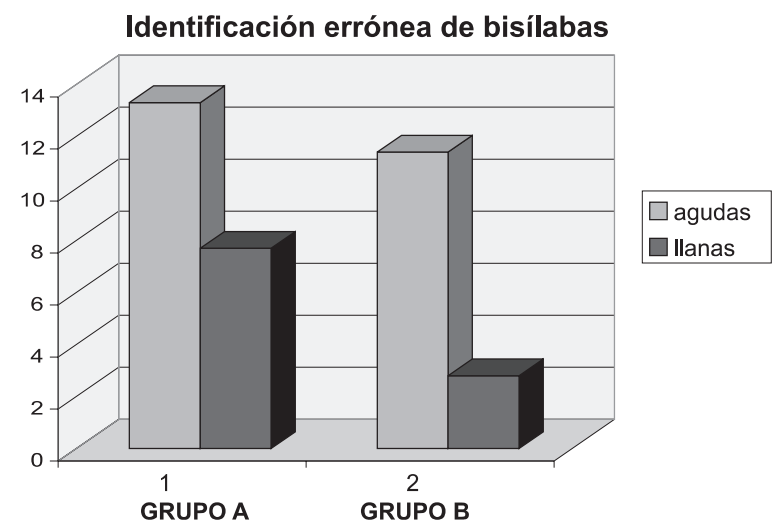

Figura 4: Los porcentajes de identificación errónea de la palabras bisílabas

Identificación errónea de trisílabas GRUPO A columnas 1,2,3 GRUPO B columnas 4,5,6

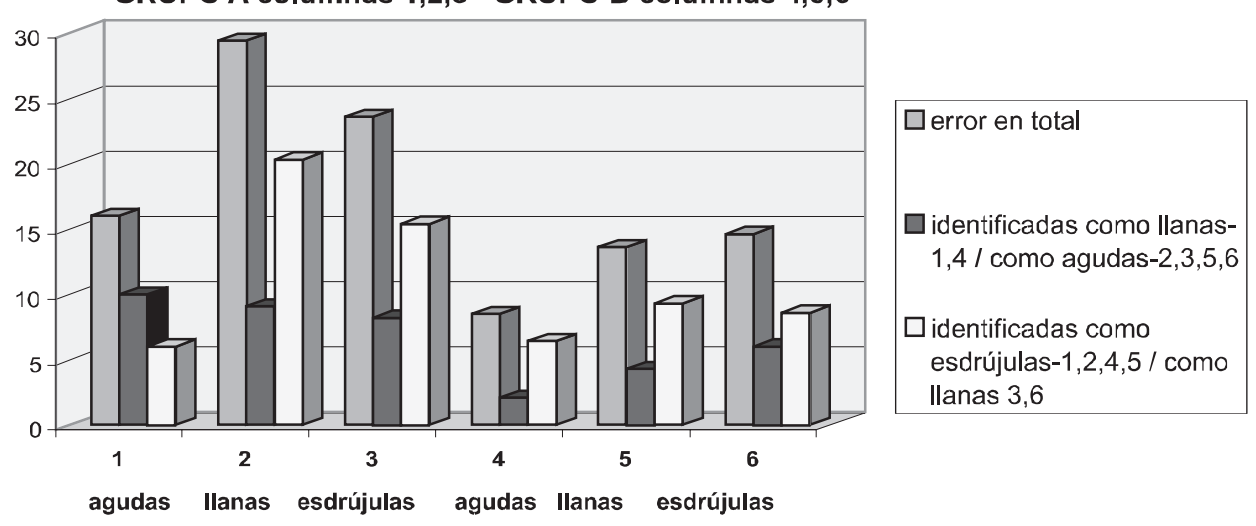

Figura 5: Los porcentajes de la identificación errónea de la palabras trisílabas 


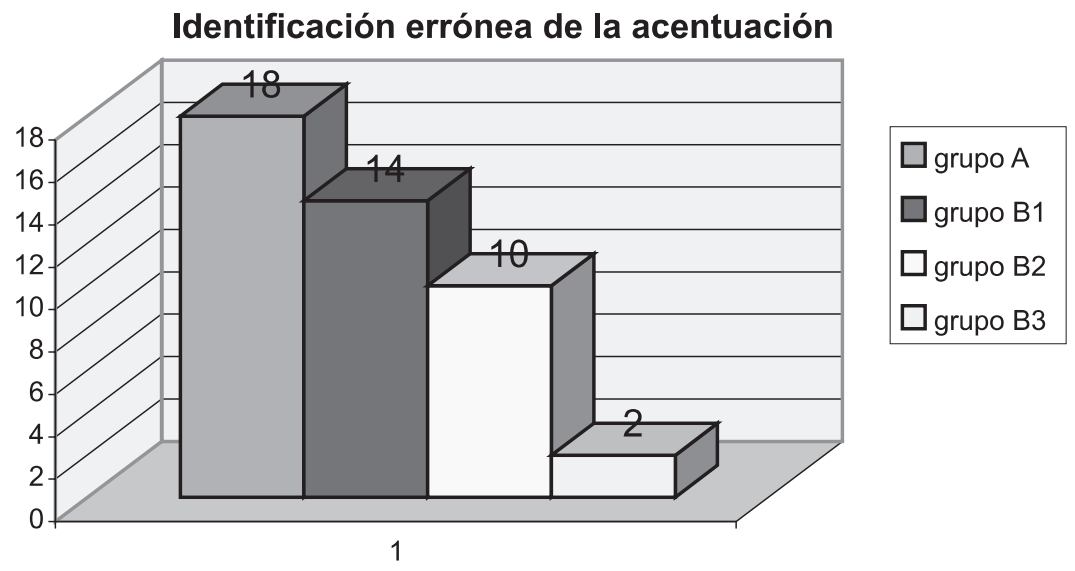

Figura 6: Comparación de los resultados de la percepción de acentuación del grupo A y B

\section{La entonación}

El peor resultado se ha conseguido con la percepción de la entonación.

Los resultados del grupo A (fig.7) demuestran que la identificación errónea de los patrones entonativos es más alta con los enunciados enfáticos, que se identifican como declarativos en el $33,3 \%$, y en el 16,1\%, como interrogativos. Esto se explica por el uso del tonema descendente en español que se interpreta como declarativo porque en esloveno, para expresar los enunciados enfáticos, prevalece el uso del tonema ascendente. Siguen las preguntas absolutas que se confunden con las enfáticas en $21,4 \%$, probablemente por la misma razón, y en el $11,9 \%$ con enunciados afirmativos. Los enunciados afirmativos se identifican con el $12,6 \%$ como enfáticos y el 7,4\% como interrogativos. Probablemente, porque español tiene el campo tonal más amplio, se puede decir que hay más diferencia en el tono entre el primer y el último pico de un grupo fónico y la flexión final es bastante inclinada. Al contrario, el esloveno tiende a una entonación neutra con las curvas melódicas poco marcadas. Lo menos problemático para los esloveno hablantes es identificar correctamente los enunciados interrogativos de preguntas pronominales, que se identifican como afirmativas en el 4,8\% y como enfáticas en el $8 \%$. Resulta que las preguntas pronominales son más fáciles de identificar porque empiezan con el pronombre interrogativo, que ayuda a identificar el enunciado como una pregunta.

Los oyentes del grupo B (fig.8) han hecho la prueba bastante mejor, es decir, que se ha comprobado que el error disminuye aumentando el tiempo de aprendizaje, pero se mantiene la correlación entre el porcentaje y el tipo de error (fig.9). Lo más interesante es que desciende en mayor grado el porcentaje de errores que llegan a los porcentajes más bajos del grupo A y que los porcentajes altos de ciertos errores bajan muy poco. Por ejemplo, los errores de identificación de las preguntas absolutas: el grupo A los han confundido con las afirmativas en $11,9 \%$ y con las enfáticas en el $21,4 \%$; el grupo B con las afirmativas tan sólo en el 2,9\% pero con las enfáticas en el 20,2\%. 


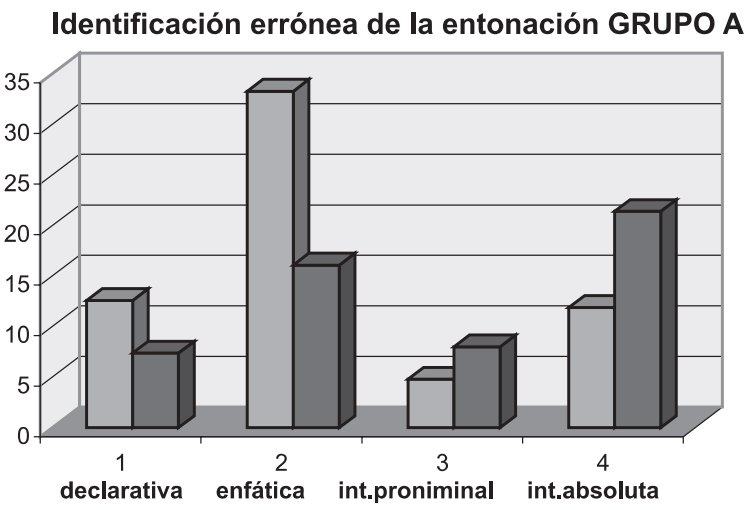
$\square$ identificada como declarativa / como enfática columna 1
$\square$ identificada como interrogativa 1,2 / como enfática 3,4

Figura 7: Los porcentajes de la identificación errónea de la entonación del grupo A
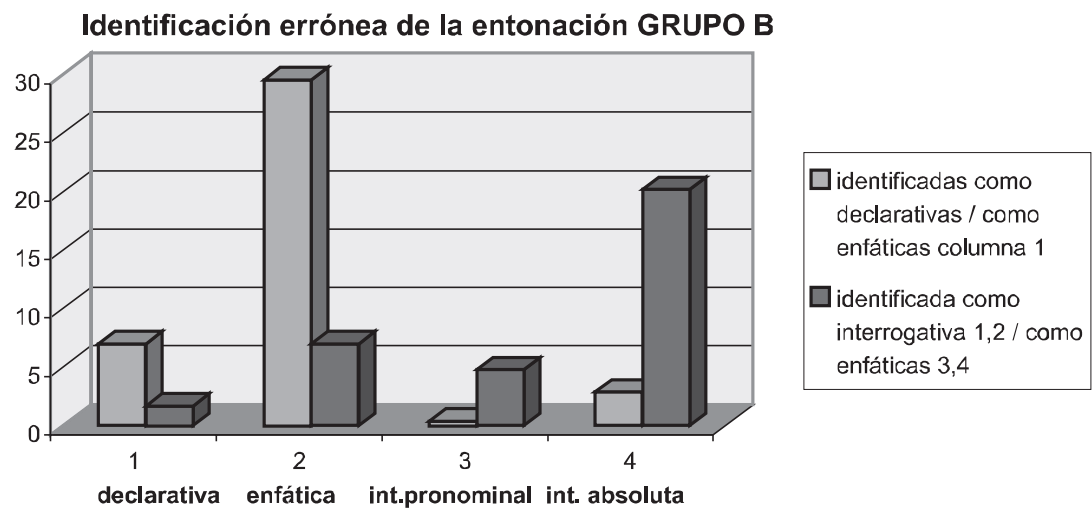

Figura 8: Los porcentajes de la identificación errónea de la entonación del grupo B

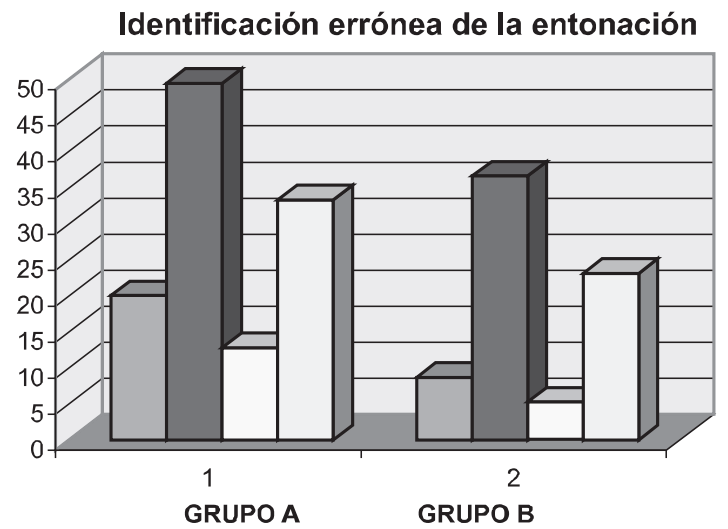

\begin{tabular}{|l|}
$\square$ declarativa \\
$\square$ enfática \\
$\square$ interrogativa \\
pronominal \\
$\square$ interrogativa absoluta
\end{tabular}

Figura 9: Los porcentajes de la identificación errónea de los patrones básicos entonativos del grupo A y B 


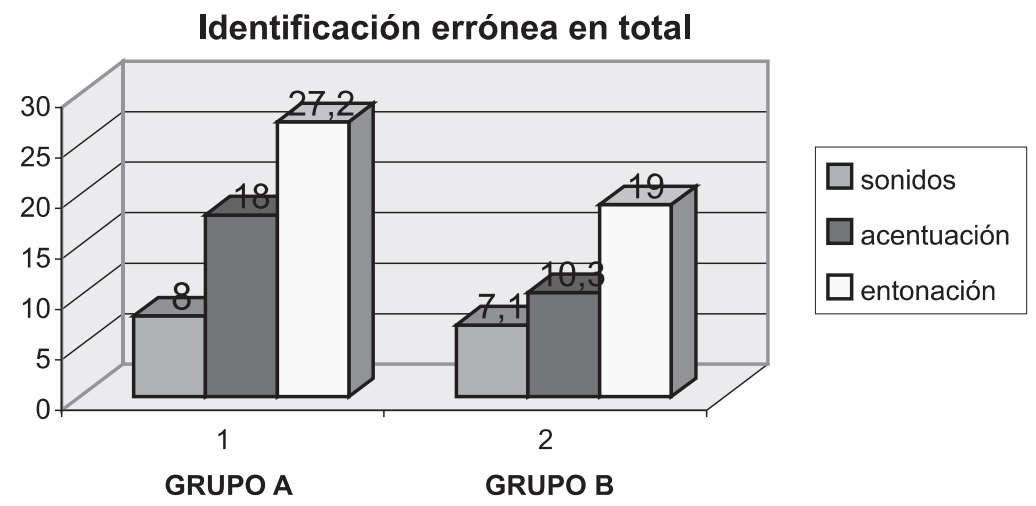

Figura 10: Comparación de la percepción de la entonación del grupo A y $B$

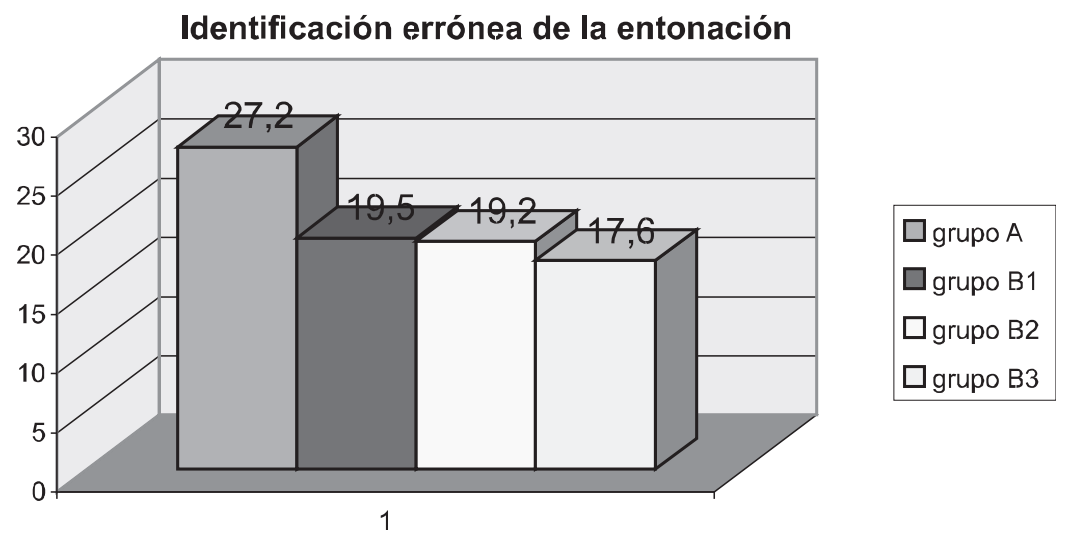

Figura 11: Comparación de los resultados de la percepción de la pronunciación del grupo A y B

\section{Conclusiones}

Los eslovenos, en general, hablan bien las lenguas extranjeras y no tienen muchas dificultades con el aprendizaje de la pronunciación. Sin embargo, existen diferencias entre el sistema fonético-fonológico esloveno y español que pueden generar la ininteligibilidad y formación del acento extranjero.

Las pruebas auditivas de percepción han demostrado que los hablantes eslovenos cometen el porcentaje más alto de identificación errónea con los rasgos fonéticos cuya realización se diferencia de un sistema a otro. Además, los resultados de las pruebas muestran que el mayor problema lo representa la identificación correcta de la entonación, seguida por la acentuación y los sonidos, cuya percepción correcta no parece ser difícil para los eslovenos (fig.11). Este hecho nos da una prueba muy importante para sostener la teoría de que no sólo los sonidos, que han sido tradicionalmente el objetivo central de la enseñanza de la pronunciación, sino que también y, sobre todo, la prosodia desempeña una papel decisivo en la pronunciación correcta y legible y debe, sin lugar a dudas, ocupar la 
mayor parte del tiempo en la enseñanza de la pronunciación. La comparación de los resultados de las pruebas del grupo A y del grupo B nos induce a otra conclusión significativa: el proceso instructivo ayuda a disminuir la percepción errónea, es decir que cuanto más larga la duración del proceso instructivo y la exposición al input en la lengua meta tanto más correcta la percepción, y consecuentemente, la producción. Esto demuestra sobre todo la percepción de la acentuación y entonación, y en un menor grado, la percepción de los sonidos.

De la comparación de los dos sistemas fonético-fonológicos esloveno y español, los resultados de las pruebas auditivas y los ejemplos de la producción de los eslovenohablantes (Gospodarič, 2003), se puede concluir que en la enseñanza de la pronunciación a eslovenohablantes debería ponerse la atención a los siguientes rasgos segmentales y suprasegmentales:

- Pronunciación de las vocales $[\alpha, \epsilon, o, v]$ con el grado intermedio de abertura,

- Los diptongos crecientes y triptongos,

- Las consonantes $[\mathrm{B}, \Gamma, \Delta, \rho]$,

- Las diferencia entre $[\vartheta]-[\mathrm{v} l]$ y $[\Delta]-[\lambda \mathrm{l}]$,

- Las consonantes $[T, \xi, \sigma]$ cuando se pretende tener la pronunciación del acento castellano-peninsular,

- Las consonantes $[\Lambda, \varphi, \mho, \tau \Sigma]$ cuando se pretende tener una pronunciación más correcta y perfecta,

- Pronunciación ortográfica de la letras «h, j, ll, ñ, z, ce, ci, ge, gi, gue, gui, que, qui»,

- La acentuación y el ritmo en general, y en particular, la acentuación de las palabras agudas y cuando esta acentuación tiene valor fonológico,

- La entonación de todo tipo de enunciados, especialmente de los enfáticos y las preguntas absolutas.

Para concluir se propone una secuenciación del sílabo para la enseñanza de la pronunciación española a hablantes eslovenos usando los niveles comunes del Marco de Referencia Europeo, que se refleja en la figura 12. No obstante, los contenidos elegidos deben ser modificados conforme a las necesidades de los alumnos y otras circunstancias que acompañan un curso particular. 


\begin{tabular}{|c|c|}
\hline NIVEL & CONTENIDOS \\
\hline $\mathrm{C} 2$ & $\begin{array}{l}\text { Refinamiento de todos los elementos de la pronunciación, acercándose lo más posible } \\
\text { a la variante estándar y coloquial hablada por los nativos. }\end{array}$ \\
\hline $\mathrm{C} 1$ & $\begin{array}{l}\text { Sonidos: reconocimiento de los rasgos típicos de las variantes del español, refinamien- } \\
\text { to de la producción de los sonidos de la variante estándar y coloquial elegida por el } \\
\text { alumno. } \\
\text { Acentuación: el ritmo del discurso en general. } \\
\text { Entonación: patrones entonativos de las frases más complejas, matices sutiles de sig- } \\
\text { nificados expresados por la entonación, sobre todo enfáticos. } \\
\text { Atención a la fluidez de todos los rasgos del discurso, la corrección de los rasgos que } \\
\text { provocan el efecto de acento extranjero. }\end{array}$ \\
\hline B2 & $\begin{array}{l}\text { Sonidos: los diptongos crecientes y triptongos, las consonantes }[\Lambda, \varphi, \vartheta, \tau \Sigma] \text {. } \\
\text { Acentuación: el uso de la tilde con monosílabas, identificación y producción del sirrema } \\
\text { y la sinalefa. } \\
\text { Entonación: patrones entonativos de las frases compuestas. } \\
\text { Atención a la fluidez de discurso en general, la corrección de los rasgos que provocan } \\
\text { el efecto de acento extranjero. }\end{array}$ \\
\hline B1 & $\begin{array}{l}\text { Sonidos: identificación y producción de los diptongos y triptongos, identificación y } \\
\text { producción de la diferencia entre }[\vartheta]-[\mathrm{V}] \mathrm{y} \Lambda]-[\lambda \mathrm{l}] \text {, y otros grupos consonánticos, } \\
\text { la producción de las consonantes }[\mathrm{B}, \Gamma, \Delta, \rho],(\mathrm{y} \text { las consonantes }[\mathrm{T}, \xi, \sigma] \text { con la vari- } \\
\text { ante castellana-peninsular). } \\
\text { Acentuación: identificación y producción de la sílaba fuerte de palabras, sobre todo las } \\
\text { agudas, el uso del acento ortográfico, identificación y producción del ritmo de la frase } \\
\text { compuesta en la cadena hablada. } \\
\text { Entonación: patrones entonativos de las frases compuestas, entonación de los enunciados } \\
\text { enfáticos y las preguntas absolutas. } \\
\text { Atención a la fluidez de las frases o discursos más largos, la corrección de los rasgos } \\
\text { que afectan la comprensibilidad e inteligibilidad. }\end{array}$ \\
\hline A2 & $\begin{array}{l}\text { Sonidos: identificación y producción de las vocales }[\mathrm{a}, \mathrm{e}, \mathrm{o}, \mathrm{u}] \text { y las consonantes }[\mathrm{B}, \Gamma \text {, } \\
\Delta, \rho] \text {, pronunciación de las letras «h, } \mathrm{j}, \mathrm{ll}, \tilde{\mathrm{n}}, \mathrm{z}, \mathrm{ce}, \mathrm{ci}, \mathrm{ge}, \text { gi, gue, gui, que, qui». } \\
\text { Acentuación: las reglas de acentuación, identificación y producción de la sílaba fuer- } \\
\text { te de palabras agudas, llanas y esdrújulas, identificación y producción del ritmo de la } \\
\text { frase en la cadena hablada. } \\
\text { Entonación: segmentación del discurso en grupos fónicos, identificación y realización } \\
\text { de los patrones básicos entonativos, sobre todo de los enfáticos y preguntas absolutas. } \\
\text { Atención a la comprensibilidad e inteligibilidad en general y a la fluidez de las frases } \\
\text { simples y discursos muy breves. }\end{array}$ \\
\hline A1 & $\begin{array}{l}\text { Sonidos: alfabeto, identificación y producción de fonemas españoles. } \\
\text { Acentuación: identificación de la sílaba fuerte de palabras agudas, llanas y esdrújulas, } \\
\text { identificación del ritmo de la frase en la cadena hablada. } \\
\text { Entonación: identificación y realización de los patrones básicos entonativos (declarativo, } \\
\text { interrogativo, enfático). } \\
\text { Atención a la comprensibilidad e inteligibilidad de la pronunciación. }\end{array}$ \\
\hline
\end{tabular}

Figura 12: La secuenciación de los contenidos de la enseñanza de la pronunciación española para eslovenohablantes 


\section{BIBLIOGRAFÍA}

Anderson-Hsieh, J.; R. Johnson; K. Koehler (1992): «The relationship between native speaker judgements of nonnative pronunciation and deviance in segmentals, prosody and syllable structure». En: Language Learning, 42, 529-555.

Castino, J. (1996): «El impacto de un curso de fonética en la adquisición de la fonología española». En: Cuadernos Cervantes, enero, 44-47.

Consejo de Europa (2001): «Marco común europeo de referencia para las lenguas: aprendizaje, enseñanza, evaluación». En: www.cvc.cervantes.es/obref/marco/. Strasbourg: Consejo de Europa.

Cortés Moreno, M. (1992): Fonología eslovena y española contrastadas y corrección fonética aplicada a eslovenohablantes. Memoria de máster. Barcelona: Universitat de Barcelona.

Derwing, T. M.; M. J. Munro; G. Wiebe (1998): «Evidence in favour of a broad framework for pronunciation instruction». En: Language Learning, 48, 393-410.

Elliot, A. R. (1997): «On the teaching and aquisition of pronunciaciation within a communicative approach». En: Hispania, 80, 95-108.

Gospodarič, K. (2003): La enseñanza de la pronunciación del español como lengua extranjera. Memoria de máster: Universidad de Salamanca.

Munro, M. J.; T. M. Derwing (1999): «Foreign accent, comprehensibility, and intelligibility in the speech of second language learners». En: Language Learning, 49. Supplement 1 (Pedagogical issues in language learning), 285-312.

Neufeld, G. (1987): «On the aquisition of prosodic and articulatory features in adult language learning». En: Interlanguage Phonology: The acquisition of a second language sound system, G. Ioup y H. Weinberg (eds.). New York: Newbury House, 337-348.

\section{POUČEVANJE IZGOVORJAVE ŠPANŠČINE KOT TUJEGA JEZIKA ZA SLOVENCE: IZHODIŠČA}

Poučevanje izgovorjave tujega jezika, kot tudi španščine, se v glavnem zanemarja v primerjavi z drugimi jezikovnimi znanji, kar se opaža v razredu, saj se je skoraj ne poučuje. Še posebej ne prozodije, ki veliko bolj vpliva na oblikovanje tujega naglasa in jasnosti kot nepravilno izgovorjeni glasovi, čeprav ravno pravilna in jasna izgovorjava predstavlja prvi korak k razumljivosti nekega pogovora in posledično h komunikaciji med govorci. Rezultati slušnih preizkusov prepoznavanja španščine za slovenske govorce so potrdili te teze, hkrati pa pa so pridobljeni podatki omogočili določitev ciljev za poučevanje izgovorjave španščine za Slovence. 